zwischen den Konzepten des ius cogens, der erga omnes-Verpflichtungen und des international crime (S. 31-48). Der weitere Aufbau des zweiten Teils wird dann jedoch schwer verständlich, da Theorien und Konzepte zu knapp erläutert werden und eine Darstellung des Verantwortlichkeitsregimes in den Stufen des primären, sekundären und tertiären Rechtsverhältnisses zunächst nicht einleuchten will. Anhand der in der Völkerrechtslehre vertretenen Theorien und den Entwürfen der ILC geht die Autorin der Frage nach, ob erga omnes-Verpflichtungen als subjektive Rechte zu qualifizieren sind oder ob es sich um .bloße, auf der subjektiven Pflichtverletzung beruhende Berechtigungen handelt (S. 56-65). Des weiteren wird das Verantwortlichkeitsregime einer erga omnes-Verletzung auch unter Berücksichtigung sogenannter Subsysteme (z.B. self-contained regimes, die die Anwendung der allgemeinen Rechtsfolgen völkerrechtswidriger Akte vollkommen ausschließen sollen) dargestellt. Unter dem Titel des tertiären Rechtsverhältnisses (S. 78-88) werden sodann die Kompetenz und die Möglichkeit der Durchsetzung von erga omnes-Verpflichtungen durch die Organe der Vereinten Nationen erläutert.

Der abschließende dritte Teil der Arbeit stellt die möglichen Rechtsquellen von Verfahrenshindernissen und Prozeßeinreden des Gegners dar. Claudia Annacker kommt zu dem positiven Schluß der Durchsetzbarkeit von erga omnes-Verpflichtungen vor dem IGH. Einschränkend stellt sie jedoch fest, "daß eine effektive und umfassende Streiterledigung von erga omnes-Verpflichtungen vor dem IGH erst dann garantiert wäre, wenn das Verfahren zu einem echten objektiven Verfahren mit einer die Gemeinschaft vertretenden Prozeßpartei gestaltet werden würde" (S. 121).

Insgesamt ist die Arbeit ein begrüßenswerter Beitrag zur Beleuchtung des umstrittenen Rechtinstitutes der erga omnes-Verpflichtungen. Fehlende Zusammenfassungen und eine verwirrende Gliederung erschweren dem Leser jedoch streckenweise die Nachvollziehbarkeit der Gedankenführung.

Birgit Schröder

\title{
Anne Peters \\ Das Gebietsreferendum im Völkerrecht. Seine Bedeutung im Licht der Staatenpraxis nach 1989
}

Schriftenreihe Europäisches Recht, Politik und Wirtschaft, Band 179

Nomos Verlagsgesellschaft, Baden-Baden 1995, 562 S., DM 128,--

Der Umbruch in Osteuropa hat das moderne Völkerrecht in Bereichen auf den Prüfstand gestellt, die sich unter Geltung der UN-Charta bis dahin in einer Art Dornröschenschlaf befunden hatten. Dies betrifft in besonderem Maße die völkerrechtlichen Regeln des Gebietwechsels, die in der Zeit nach dem 2. Weltkrieg allenfalls die sich aus der Dekolonisierung ergebenen Probleme zu verarbeiten hatten, seit 1989 aber mit einer Vielzahl von 
Staatsgründungen und territorialen Neugliederungen konfrontiert werden. Die bei Schwarze in Freiburg entstandene Dissertation spürt dem daraus für die überkommenen Regeln resultierenden Veränderungsdruck nach, und zwar für einen Teilbereich des Völkerrechts, der bislang mit dem Begriff "Plebiszittheorie" schlagwortartig gekennzeichnet war. Sie fragt, ob sich durch die Entwicklungen seit 1989 ein Satz des Völkergewohnheitsrechts herausgebildet hat, wonach Gebietsveränderungen zwingend an ein Referendum unter der betroffenden Bevölkerung geknüpft sind.

Die Arbeit ist nach einem einleitenden Abschnitt mit einem historischen Rückblick in zwei Hauptteile gegliedert, von denen der erste induktiv die Staatenpraxis im Zeitraum zwischen Ende 1990 und Sommer 1994 untersucht, während der zweite quasi deduktiv eine völkerrechtssystematische Einbindung der Abstimmungspflicht in übergeordnete Prizipien wie Selbstbestimmung und Demokratie versucht.

Schon bei der Entfaltung der Fragestellung (S. 30-49) relativiert die Verfasserin den vermeintlich klaren Titel ihrer Arbeit, der auf den völkerrechtlichen Gebietswechsel, also den Übergang der territorialen Souveränität zielt (so zunächst auch die begriffliche Klärung auf S. 30). Wenn aber dann mit dem plakativen Argument, die Völkerrechtsentwicklung mache nicht an den Staatsgrenzen halt, der Untersuchungsgegenstand auf das innerstaatliche Autonomiereferendum ausgedehnt wird (S. 40 f.), so verschwimmt die Stoßrichtung der Abhandlung insgesamt: Natürlich wirken völkerrechtliche Gewährleistungen von Menschen- und Minderheitenrechten vor allem im Staatsinnern, mit der völkerrechtlichen Zuordnung von Territorium aber hat dies nichts mehr zu tun. Vor allem da das Augenmerk der Verfasserin auf die Herleitung neuer Gewohnheitsrechtssätze gerichet ist, hätte bereits bei der Eingrenzung des Untersuchungsgegenstandes die Beschränkungs auf ein Referendumsziel - innerstaatliche Autonomie oder völkerrechtlicher Gebietswechsel - nahegelegen. Diese Beschränkung nimmt die Verfasserin dann inkonsequenterweise vor, wenn sie den in Staatenpraxis und Rechtsüberzeugung nachzuweisenden Satz als Postulat formuliert: "Jede nicht rein innerstaatliche Gebietsveränderung bedarf eines Referendums" (S. 79). Bei der Untersuchung der einzelnen Beispiele aus der Staatenpraxis aber werden die innerstaatlichen Automiereferenden dennoch miteinbezogen, so daß der Leser über das genaue wissenschaftliche Ziel der Untersuchung letztlich im unklaren bleibt.

Ein kurzer historischer Abschnitt (S. 49-74) faßt vor allem die Plebiszitpraxis nach dem 1. Weltkrieg'sowie in der Epoche der Deklonisierung zusammen und kommt zu dem der ganz herrschenden Meinung entsprechenden Ergebnis, daß Referenden zu Gebietsveränderungen bis 1990 allenfalls aus Gründen der politischen Opportunität, nicht aber in Erfüllung einer Rechtspflicht stattfanden.

Der erste Schwerpunkt der Arbeit bringt eine umfassende Darstellung der Referenden über Gebiets-oder Statusveränderungen, die in der fraglichen Zeit stattgefunden haben (S. 85308). Naturgemäß stehen hier die Zerfallsprozesse in Jugoslawien und der Sowjetunion im Vordergrund, die nach Ansicht der Verfasserin jedoch beide für das Bestehen einer gefestigten Rechtsüberzeugung im Sinne eines völkerrechtlichen Referendumsgebots nicht genügend Anhaltspunkte hergeben (S. 136 bzw. 225). Gleiches gilt unter dem Strich für die 
ebenfalls erörterten Referenden in Eritrea, Kanada und Puerto Rico. Die ohne Volksabstimmung vorgenommenen Gebietsveränderungen, die für die Frage nach dem Bestehen des postulierten Rechtssatzes sehr viel aussagekräftiger gewesen wären, werden sehr kurz und mit dem Verweis auf rechtliche Besonderheiten recht geringschätzig abgehandelt ( $S$. 264-279). Insgesamt erbringt die Sichtung der Staatenpraxis dennoch das - angesichts der Unterschiedlichkeit der behandelten Fälle kaum überraschende - Ergebnis, daß weder eine einheitliche Praxis noch eine ausreichende Rechtsüberzeugung für die Geltung eines völkerrechtlichen Referendumserfordernisses wirklich schon nachweisbar sind (S. 316317). Doch auch die Aussage, man befinde sich insoweit im " Stadium der Rechtswerdung", wird durch die vorangegangene Sichtung des relevanten Materials nicht getragen. Es ist daher nur schwer nachvollziehbar, wenn die Verfasserin am Ende des induktiven Teils das Problem auf die Frage nach dem Entstehungszeitpunkt der neuen völkerrechtlichen Regel verschiebt, die Normbildung als solche mithin bereits für unabweisbar hält.

Der Anfang des nächsten Hauptteils resümiert den vorangegangenen mit den Worten, es habe "nicht ohne Einschränkungen" festgestellt werden können, "daß ein neues völkerrechtliches Abstimmungserfordernis besteht" (S. 319). Der Leser ist verwirrt, hat er doch das gerade gelesene Ergebnis der induktiven Prüfung anders in Erinnerung. Im folgenden untenimmt die Verfasserin nun den Versuch, diese "Vorgabe" in die Grundprinzipien des allgemeinen Völkerrechts rechtssystematisch einzubinden. Ansatzpunkt dafür ist zunächst das Selbstbestimmungsrecht der Völker, das auch auf die Stellung von Minderheiten und Volksgruppen im Innern eines Staates bezogen wird (S. 335-342). Die alte Frage nach dem aus dem Selbstbestimmungsrecht resultierenden Recht auf Sezession läßt die Verfasserin offen, statt dessen statuiert sie aus rechtspolitischen Gründen die Abhaltung eines Sezessionsreferendums als Verfahrensvoraussetzung für die völkerrechtliche Rechtmäßigkeit der Sezession (S. 361-364). Dieser "Setzung" entsprechend werden zum Träger des zum Abstimmungsrecht geronnenen Selbstbestimmungsrechts die Bewohner des betroffenden Gebiets bestimmt (S. 376-377).

Zur Absicherung ihrer These untersucht die Verfasserin anschließend die Geltung eines Demokratiegebots im Völkergewohnheitsrecht (S. 396-432), die sie sowohl als Satz des Völkergewohnheitsrechts als auch als allgemeinen Rechtsgrundsatz für möglich hält. Das Ergebnis bleibt offen, unter selektivem Rückgriff auf einige "soft-law"-Dokumente wird aber ein "untechnischer Einfluß des Demokratiegrundsatzes auf die Völkerrechtsentwicklung" (S. 420) sowie ein "völkerrechtlicher Demokratietrend" (S. 432) konstatiert, der belegen soll, daß sich ein Abstimmungsgebot vor Gebietsveränderungen in die gegenwärtige Völkerrechtsordnung einfügt. Schließlich wird auch die zwangsläufige Durchbrechung des den völkerrechtlichen Staatsbegriff bestimmenden Effektivitätsprinzips durch das statuierte Verfahrenserfordernis für Gebietsveränderungen als unproblematisch eingestuft, wenngleich vor weiteren Auf weichungen durch Anforderungen demokratischer Legitimität gewarnt wird (S. 437-458).

Ihre "Auslegung des Selbstbestimmungsrechts im Sinne eines demokratisch fundierten Rechts" (S. 470) bringt die Verfasserin zu der Schlußfolgerung, daß die demokratische 
Entscheidung "eine notwendige, jedoch keine hinreichende Bedingung der Völkerrechtsgemäßheit einer territorialen Veränderung, insbesondere einer Sezession" sei (S. 474). Sogar die Einordnung als erga omnes-Verpflichtung sei "sinnvoll" (S. 476). Für die Rechtsfolgen einer Verletzung des Abstimmungserfordernisses nimmt die Verfasserin die zutreffende Unterscheidung zwischen völkerrechtlicher Rechtswidrigkeit und Nichtigkeit vor und befürwortet nach dem Vorbild der sog. Stimson-Doktrin eine Nichtanerkennungspflicht dritter Staaten (S. 481-486).

Insgesamt handelt es sich um eine Untersuchung mit zwei auch in ihrer dogmatischen Stringenz sehr unterschiedlichen Teilen. Der induktive Haupteil bringt eine detaillierte und anschauliche Aufarbeitung des relevanten Praxismaterials und ist als solcher von bleibendem wissenschaftlichen Wert. Der Blick für eine nüchterne Wertung dieser Staatenpraxis scheint der Verfasserin allerdings durch ihr dogmatisches Vorverständnis verstellt zu sein. Dieses zeigt sich im "deduktiven" Hauptteil, der aus abstrakten Prinzipien mit rechtspolitischen Erwägungen eine bindende völkerrechtliche Verfahrensnorm ableitet, die mit den Strukturen des geltenden Völkerrechts nur schwer zu vereinbaren ist. Wie sehr dies Ergebnis zudem an den Realitäten der Staatenpraxis vorbeigeht, zeigt nicht zuletzt die jüngst erzielte Friedensregelung für Bosnien-Herzegowina, durch die mit Billigung der gesamten Staatengemeinschaft (universelle Rechtsüberzeugung!) erhebliche territoriale Neugliederungen vorgenommen wurden, ohne daß jemand nach dem Willen der betroffenen Bevölkerung gefragt hätte.

Oliver Dörr

\section{Mohamed Munavvar}

Ocean State - Archipelagic Regimes in the Law of the Sea

Publications on Ocean Development, Vol. 22

Martinus Nijhoff Publishers, Dordrecht, 1995, 225 S., $£ 74.00$

Zu den neuen Konzepten des UN-Seerechtsübereinkommens (SRÜ) von 1982 (bisher für 81 Staaten in Kraft getreten) zählen neben der 200 Seemeilen-Wirtschaftszone, dem Tiefsee-Regime und der Streitregelung auch das Archipel-Konzept, dem diese Monographie gewidmet ist.

Munavver unterscheidet drei Typen von Archipelen: erstens "Küstenarchipele", d.h. Inselketten oder -gruppen vor einer Festlandsküste (z.B norwegische Schären), zweitens "Ozeanische Archipele" von Küstenstaaten (z.B.Galapagos/Equador) und drittens "Archipelstaaten"(wie Indonesien oder Fiji), also Staaten, die vollständig aus Inseln bestehen.

Die Kapitel 1 bis 3 dokumentieren die völkerrechtliche Entwicklung dieser archipelagischen Regime anhand der neueren Seerechtsentwicklung, wobei das Konzept der "Archipelstaaten" und seine Umsetzung in einem eigenen Abschnitt IV des SRÜ in den Artikeln 